\title{
ASYMPTOTIC EXPANSION OF A SERIES OF RAMANUJAN
}

\author{
by BRUCE C. BERNDT* and RONALD J. EVANS
}

(Received 1st May 1990)

An asymptotic expansion is given for the series

$$
\sum_{n=0}^{\infty} \frac{\Gamma(n+s)}{n !} \frac{(a+n)^{n-r}}{(x+a+n)^{n+s}}
$$

as $x \rightarrow \infty$ in the sector $|\operatorname{Arg} x| \leqq \pi / 2-\delta$. Here $\delta, \operatorname{Re}(a)$, and $\operatorname{Re}(s)$ are positive and $r$ is a positive integer. In the case $a=r=s=1$, this yields the nontrivial result

$$
e^{x} \sum_{k=1}^{\infty} \frac{1}{k^{2}(1+x / k)^{k}}-\frac{e^{x}}{x}=-\frac{2}{x^{2}}+\frac{16}{3 x^{3}}-\frac{56}{3 x^{4}}+\frac{3712}{45 x^{5}}+0\left(\frac{1}{x^{6}}\right)
$$

stated by Ramanujan in his notebooks [6].

1980 Mathematics subject classification (1985 Revision). 41A60.

\section{Introduction}

The primary object of this paper is to prove Theorem 1 below, which gives an asymptotic expansion as $x \rightarrow \infty$ in the sector $|\operatorname{Arg} x| \leqq \pi / 2-\delta$ for the series

$$
T(x):=\sum_{n=0}^{\infty} \frac{\Gamma(n+s)}{n !} \frac{(a+n)^{n-r}}{(x+a+n)^{n+s}}
$$

where here and in the sequel $\delta>0$ is fixed and arbitrarily small, $r$ is a fixed positive integer, and $a$ and $s$ are fixed complex numbers with positive real parts.

Theorem 1 Let $N$ be an integer with $N \geqq 1$. Then as $x \rightarrow \infty$ in the sector $|\operatorname{Arg} x| \leqq \pi / 2-\delta$,

$$
T(x)=\sum_{k=0}^{r-1} A_{k} x^{-k-s}-e^{-x}\left(\sum_{m=0}^{N-1} \frac{C_{m}(x)}{(a+x / 2)^{m+1}}+0\left(x^{-1-r-N / 2}\right)\right)
$$

* Partially supported by NSF grant DMS-8820680. 
where

$$
A_{k}=\sum_{j=0}^{k}(-1)^{k-j} \Gamma(s+k)\left(\begin{array}{l}
k \\
j
\end{array}\right)(a+j)^{k-r}
$$

and where the functions $C_{m}(x)$ (defined in (2.9)) have the estimate

$$
C_{m}(x)=0\left(x^{[m / 2]-r}\right)
$$

Observe that (1.2) is a genuine asymptotic expansion, in view of (1.4). Note also that $x$ can be replaced by $x+b$ in (1.2), for any constant $b$. Thus, e.g., if the sign of $a$ is reversed in the denominator of $(1.1)$, then $C_{m}(x) /(a+x / 2)^{m+1}$ is replaced by $2^{m+1} C_{m}(x-2 a) / x^{m+1}$.

Theorem 1 was inspired by Ramanujan, who stated the case $r=s=1$ in the unorganized pages of his second notebook [6, p. 272, eq. (5)]. Ramanujan found that for $r=s=1, C_{0}(x)=x^{-1}$ and each $C_{m}(x)$ with $m \geqq 1$ is a polynomial in $x$ such that, for $k \geqq 1$,

$$
C_{2 k-1}(x)=0
$$

and

$$
C_{2 k}(x)=\left(-\frac{1}{12}\right)^{k} \frac{(2 k) !}{k !} x^{k-1}-\cdots-\frac{(2 k+1)}{2} B_{2 k} x-B_{2 k}
$$

where $B_{0}, B_{1}, B_{2}, \ldots$ are the Bernoulli numbers defined by the generating function $[1$, p. 804]

$$
\frac{t}{e^{t}-1}=\sum_{m=0}^{\infty} \frac{B_{m}}{m !} t^{m}, \quad|t|<2 \pi
$$

In fact, he computed the first five leading coefficients and the last six trailing coefficients of the polynomials $C_{m}(x)(m \geqq 1, r=s=1)$; see [6, pp. 272-273], [2]. Ramanujan [6, p. 271, eq. (2)] also stated (in a different form) the following:

Corollary 2. As $x \rightarrow \infty$ with $|\operatorname{Arg} x| \leqq \pi / 2-\delta$,

$$
\sum_{k=1}^{\infty} \frac{k^{k-2}}{(k+x)^{k}}=\frac{1}{x}-e^{-x}\left(\frac{2}{x^{2}}-\frac{16}{3 x^{3}}+\frac{56}{3 x^{4}}-\frac{3712}{45 x^{5}}+0\left(x^{-6}\right)\right)
$$

Proof. This follows from Theorem 1 with $a=r=s=1$ and $N=8$, upon substitution of the values 


$$
C_{2}=-\frac{1}{6}, \quad C_{4}=\frac{x}{12}+\frac{1}{30}, \quad C_{6}=-\frac{5 x^{2}}{72}-\frac{x}{12}-\frac{1}{42}
$$

given, e.g., by (1.6).

Writing the first few terms of the expansion in Theorem 1 in more explicit form, we obtain the following generalization of Corollary 2 :

Corollary 3. As $x \rightarrow \infty$ with $|\operatorname{Arg} x| \leqq \pi / 2-\delta$,

$$
\begin{aligned}
& \sum_{n=0}^{\infty} \frac{(a+n)^{n-1}}{(x+a+n)^{n+1}}=\frac{1}{a x}-e^{-x} \\
& \quad \times\left(\frac{2}{x^{2}}-\frac{(12 a+4)}{3 x^{3}}+\frac{\left(24 a^{2}+24 a+8\right)}{3 x^{4}}-\frac{\left(720 a^{3}+1440 a^{2}+1200 a+352\right)}{45 x^{5}}+0\left(x^{-6}\right)\right)
\end{aligned}
$$

In Corollaries 2 and 3, the asymptotic series are expressed explicitly in descending powers of $x$. The general asymptotic series in Theorem 1 could also be expressed in this way if an asymptotic expansion could be given for each $C_{m}(x)$ in descending powers of $x$. This is indeed possible and we show how this can be accomplished in Section 6. If $s$ is a positive integer, we prove the stronger result that $C_{m}(x)$ is a Laurent polynomial in $x$.

Ramanujan [6, p. 270, eq. (1)] also found the following interesting exact formula for the series in Corollary 2:

$$
\sum_{k=1}^{\infty} \frac{k^{k-2}}{(x+k)^{k}}=\frac{1}{x}+e^{-x}\left(-\frac{1}{x}+\sum_{k=1}^{\infty} k^{k-2} e^{-k} \sum_{j=1}^{k} \frac{(x+k)^{-j}}{(k-j) !}\right)
$$

where $\operatorname{Re}(x)>0$. For a proof, see [2].

In Section 2, we discuss confluent hypergeometric functions and introduce further notation. The goal of Section 3 is to prove the integral representation (3.8) for $T(x)$. In Section 4, we prove Lemma 4, which provides bounds for the derivatives with respect to $t$ of the function $f(t, x)$ defined in (2.8). The proof of Theorem 1 is given in Section 5 and is based on the results of the previous sections. Finally, in Section 6, we show that $C_{m}(x)$ possesses an asymptotic expansion in descending powers of $x$, and that moreover $C_{m}(x)$ is a Laurent polynomial in $x$ when $s$ is an integer.

\section{Confluent hypergeometric functions}

Consider the confluent hypergeometric function

$$
{ }_{1} F_{1}(s, s+r ; z)=\sum_{m=0}^{\infty} \frac{(s)_{m} z^{m}}{(s+r)_{m} m !}, \quad|z|<\infty,
$$


with the usual notation

$$
(s)_{m}=\Gamma(s+m) / \Gamma(s), \quad m \geqq 0 .
$$

This function is related to $U(s, s+r ; z)$, the confluent hypergeometric function of the second kind, by

$$
\begin{aligned}
{ }_{1} F_{1}(s, s+r ; z)= & \frac{\Gamma(s+r)}{\Gamma(r)} e^{i \pi s} U(s, s+r ; z) \\
& +\frac{\Gamma(s+r)}{\Gamma(s)}(-1)^{r} e^{2} U(r, s+r ;-z), \quad \frac{\pi}{2}<\arg z<\frac{3 \pi}{2}
\end{aligned}
$$

see $[5$, p. 257 , eq. (10.09)], [4, p. 270, eq. (9.12.4)]. In many books (e.g., [4, p. 263]), $U$ is designated by $\Psi$. As $z \rightarrow \infty$ with $|\arg z| \leqq 3 \pi / 2-\delta$, we have the asymptotic expansion $[5$, p. 256]

$$
U(r, s+r ; z) \sim \sum_{m=0}^{\infty} \frac{(-1)^{m}(r)_{m}(1-s)_{m}}{m ! z^{m+r}}
$$

Since $r$ is a positive integer, $U(s, s+r ; z)$ can be expressed as a Laguerre polynomial; see $[3$, p. 189 , eq. (14)], [3, p. 188 , eq. (7)]. Thus

$$
U(s, s+r ; z)=\sum_{k=0}^{r-1} \frac{(-1)^{k}(s)_{k}(1-r)_{k}}{z^{k+s}} .
$$

For brevity, write, for $t \geqq 0$,

$$
w=w(t)=t /\left(1-e^{-t}\right)
$$

so that by (1.7),

$$
w=\sum_{m=0}^{\infty} \frac{B_{m}}{m !}(-t)^{m}, \quad|t|<2 \pi .
$$

For $t \geqq 0, \operatorname{Re}(x)>0$, define

$$
f(t, x)=e^{x(1-w+t / 2)}(-t)^{r-1} w^{s} U(r, s+r ; w x) .
$$

Finally, the functions $C_{m}(x)$ in Theorem 1 are defined by

$$
C_{m}(x)=f^{(m)}(0, x), \quad \operatorname{Re}(x)>0,
$$

where the superscript $m$ denotes the $m$ th derivative with respect to $t$. 
We remark that in the case $r=1$,

$$
f(t, x)=x^{-s} e^{x+x t / 2} \Gamma(s, w x)
$$

for the incomplete gamma function

$$
\Gamma(s, z)=\int_{z}^{\infty} e^{-t} t^{s-1} d t, \quad \operatorname{Re} s>0 .
$$

This follows from (2.8) and the formula [3, p. 136, eq. (15)]

$$
\Gamma(s, z)=e^{-z} z^{s} U(1, s+1 ; z)
$$

3. Integral representation of the series $T(x)$

Define, for each integer $m \geqq 0$,

$$
\mu_{m}=\sum_{n=0}^{\infty} \frac{\Gamma(m+s+n)}{n !(a+n)^{m+s+r}}
$$

From Euler's integral representation of the gamma function,

$$
\frac{1}{(a+n)^{m+s+r}}=\frac{1}{\Gamma(m+s+r)} \int_{0}^{\infty} e^{-n t-a t} t^{m+s+r-1} d t .
$$

Thus

$$
\mu_{m}=\frac{\Gamma(m+s)}{\Gamma(m+s+r)} \int_{0}^{\infty} e^{-a t} t^{m+s+r-1} \sum_{n=0}^{\infty} \frac{\Gamma(m+s+n)}{\Gamma(m+s) n !} e^{-t n} d t
$$

where absolute convergence justifies the interchange of integration and summation. The sum on $n$ in (3.3) equals $\left(1-e^{-t}\right)^{-m-s}$, and so

$$
\mu_{m}=\frac{\Gamma(m+s)}{\Gamma(m+s+r)} \int_{0}^{\infty} e^{-a t} t^{r-1} w^{m+s} d t
$$

where $w$ is defined in (2.6).

Recall that $T(x)$ is defined in (1.1) for $\operatorname{Re} x>0$. Assuming for the moment that $|x|<|a|$, we find that

$$
T(x)=\sum_{n=0}^{\infty} \frac{\Gamma(n+s)}{n !(a+n)^{s+r}}\left(1+\frac{x}{a+n}\right)^{-n-s}
$$




$$
=\sum_{n=0}^{\infty} \frac{\Gamma(n+s)}{n !(a+n)^{s+r}} \sum_{m=0}^{\infty} \frac{\Gamma(m+s+n)}{m ! \Gamma(n+s)}\left(\frac{-x}{a+n}\right)^{m} .
$$

By (3.1) and (3.5),

$$
T(x)=\sum_{m=0}^{\infty} \frac{(-x)^{m}}{m !} \mu_{m}, \quad|x|<|a|
$$

where absolute convergence justifies the interchange of summations. Ramanujan [6, p. 271, eq. (3)] gave the case $r=s=1$ of (3.6).

Put (3.4) in (3.6) to obtain for $|x|<|a|$,

$$
\begin{aligned}
T(x) & =\sum_{m=0}^{\infty} \frac{(-x)^{m}}{m !} \frac{\Gamma(m+s)}{\Gamma(m+s+r)} \int_{0}^{\infty} e^{-a t} t^{r-1} w^{m+s} d t \\
& =\int_{0}^{\infty} e^{-a t} t^{r-1} w^{s} \sum_{m=0}^{\infty} \frac{(-x w)^{m}}{m !} \frac{\Gamma(m+s)}{\Gamma(m+s+r)} d t,
\end{aligned}
$$

where the interchange of integration and summation can be justified by absolute convergence. By (2.1), (2.2), and (3.7),

$$
T(x)=\frac{\Gamma(s)}{\Gamma(s+r)} \int_{0}^{\infty} e^{-a t} t^{r-1} w_{1}^{s} F_{1}(s, s+r ;-w x) d t
$$

for $|x|<|a|$.

As $x \rightarrow \infty$ with $|\operatorname{Arg} x| \leqq \pi / 2-\delta,-w x \rightarrow \infty$ with $\pi / 2+\delta \leqq \arg (-w x) \leqq 3 \pi / 2-\delta$. Thus by (2.3) $-(2.5)$, the integral in (3.8) is convergent and analytic in each variable $a, x$ in the right half plane. From (1.1), $T(x)$ is also seen to be analytic in each of $a, x$ in the right half plane. Thus (3.8) holds for all $x$ with $\operatorname{Re} x>0$.

\section{Bounds for derivatives of $f(t, x)$}

The proof of Lemma 4 below makes heavy use of Faa di Bruno's formula $[7$, p. 36$] ;[8]$,

$$
\frac{d^{n}}{d t^{n}} h(g(t))=\sum \frac{n ! h_{k}(g(t))}{k_{1} ! \ldots k_{n} !}\left(\frac{g_{1}}{1 !}\right)^{k_{1}} \ldots\left(\frac{g_{n}}{n !}\right)^{k_{n}}
$$

where the sum is over all integers $k_{1}, k_{2}, \ldots, k_{n}$ for which

$$
n=k_{1}+2 k_{2}+\cdots+n k_{n}, \quad k_{i} \geqq 0,
$$


and where $k=k_{1}+\cdots+k_{n}$,

$$
h_{k}(z)=\frac{d^{k}}{d z^{k}} h(z), \quad \text { and } \quad g_{i}=g_{i}(t)=\frac{d^{i}}{d t^{i}} g(t) .
$$

Lemma 4. Fix $N \geqq 1$. As $x \rightarrow \infty$ with $|\operatorname{Arg} x| \leqq \pi / 2-\delta$,

$$
f^{(N)}(t, x)=0\left(x^{-r+[N / 2]} \sum_{j=0}^{N}|x t|^{j}\right),
$$

uniformly for $t \in[0,1]$.

Proof. Let $0 \leqq t \leqq 1$ and $n \geqq 0$. We will obtain uniform estimates for $n$th derivatives of each factor $(-t)^{r-1}, w^{s}, e^{x(1-w+t / 2)}$, and $U(r, s+r ; w x)$ of $f(t, x)$ in $(2.8)$, and then combine them to deduce (4.4) from Leibniz's rule.

First, for each $n \geqq 0$,

$$
\frac{d^{n}}{d t^{n}}(-t)^{r-1}=0(1)
$$

since $r$ is a positive integer. Next, by (2.7), we have, for each $k \geqq 0$,

$$
\frac{d^{k}}{d t^{k}} w=0(1)
$$

Consequently, by (4.1) with $h(z)=z^{s}$ and $g(t)=w$,

$$
\frac{d^{n}}{d t^{n}} w^{s}=0(1)
$$

For $|\operatorname{Arg} z| \leqq \pi / 2-\delta, U(r, s+r ; z)$ is analytic (see [5, p. 257, eq. (10.04)]) and so by $[5$, pp. 9,10 , Theorem 4.2$]$ we can differentiate in (2.4) to obtain, for $k \geqq 0$ and large $|z|$,

$$
\frac{d^{k}}{d z^{k}} U(r, s+r ; z) \sim \sum_{m=0}^{\infty} \frac{(r)_{m+k}(-1)^{m+k}(1-s)_{m}}{m ! z^{m+r+k}}=0\left(z^{-k-r}\right)
$$

Now apply (4.1) with $h(z)=U(r, s+r ; z)$ and $g(t)=w x$ to deduce from (4.6) and (4.8) that as $x \rightarrow \infty$ with $|\operatorname{Arg} x| \leqq \pi / 2-\delta$,

$$
\frac{d^{n}}{d t^{n}} U(r, r+s ; w x)=0\left(x^{-r}\right)
$$

uniformly for $0 \leqq t \leqq 1$.

A final application of (4.1) with $h(z)=e^{2 x}$ and $g(t)=(1+t / 2-w)$ yields 


$$
\frac{d^{n}}{d t^{n}} e^{x(1+t / 2-w)}=e^{x g(t)} \sum B\left(k_{1}, \ldots, k_{n}\right) g_{1}^{k_{1}} \ldots g_{n}^{k_{n}} x^{k_{1}+\cdots+k_{n}}
$$

where the sum is over integers $k_{i}$ satisfying (4.2), where the coefficients $B\left(k_{1}, \ldots, k_{n}\right)$ are independent of $x, t$, and where $g_{i}$ is defined by (4.3). By (2.7),

$$
g(t)=-\sum_{m=1}^{\infty} \frac{B_{2 m}}{(2 m) !} t^{2 m}
$$

and so

$$
g_{i}=O(t) \quad \text { for all odd } i \geqq 1
$$

and

$$
g_{j}=0(1) \text { for all } j \geqq 1 \text {. }
$$

Since $g(t) \leqq 0$ for $0 \leqq t \leqq 1$,

$$
e^{x g(t)}=0(1)
$$

By (4.2),

$$
k_{2}+k_{4}+k_{6}+\cdots \leqq \frac{1}{2}\left(k_{1}+2 k_{2}+\cdots+n k_{n}\right)=n / 2
$$

Combining (4.10) and (4.12)-(4.15), we see that

$$
\begin{aligned}
\frac{d^{n}}{d t^{n}} e^{x(1+t / 2-w)} & \ll \sum|x|^{k_{1}+k_{2}+\cdots+k_{n}} t^{k_{1}+k_{3}+k_{5}+\cdots} \\
& \ll \sum|x t|^{k_{1}+k_{3}+k_{5}+\cdots}|x|^{k_{2}+k_{4}+k_{6}+\cdots} \ll x^{[n / 2]} \sum_{i=0}^{n}|x t|^{i} .
\end{aligned}
$$

The result now follows from (4.5), (4.7), (4.9), (4.16) and Leibniz's rule.

\section{Proof of Theorem 1}

By (2.3) and (3.8),

$$
T(x)=A(x)-B(x)
$$

where 


$$
A(x)=\frac{\Gamma(s)}{\Gamma(r)} \int_{0}^{\infty} e^{-a t} t^{r-1}(-w)^{s} U(s, s+r ;-w x) d t
$$

and

$$
B(x)=\int_{0}^{\infty} e^{-a t}(-t)^{r-1} w^{s} e^{-w x} U(r, s+r ; w x) d t
$$

with $\pi / 2<\arg (-w x)<3 \pi / 2$. We first examine $A(x)$, which yields the dominant part of the asymptotic expansion of $T(x)$. Using (2.5) in (5.2), we find that

$$
\begin{aligned}
A(x) & =\frac{\Gamma(s)}{\Gamma(r)} \sum_{k=0}^{r-1}(-1)^{k}(s)_{k}(1-r)_{k} \int_{0}^{\infty} e^{-a t} t^{-1}(-w)^{s}(-w x)^{-s-k} d t \\
& =\frac{\Gamma(s)}{\Gamma(r)} \sum_{k=0}^{r-1} \frac{(-1)^{k}(s)_{k}(1-r)_{k}}{x^{s+k}} \int_{0}^{\infty} e^{-a t} t^{r-k-1}\left(e^{-t}-1\right)^{k} d t \\
& =\frac{\Gamma(s)}{\Gamma(r)} \sum_{k=0}^{r-1} \sum_{j=0}^{k} \frac{(-1)^{j}(s)_{k}(1-r)_{k}}{x^{s+k}}\left(\begin{array}{c}
k \\
j
\end{array}\right) \int_{0}^{\infty} e^{-t(a+j)} t^{r-k-1} d t,
\end{aligned}
$$

where we have expanded $\left(e^{-t}-1\right)^{k}$ by the binomial theorem. It follows easily from (5.4) that

$$
A(x)=\sum_{k=0}^{r-1} A_{k} x^{-k-s}
$$

in agreement with (1.2) and (1.3).

Now, (1.4) follows by putting $t=0$ in (4.4). Thus, by (1.2), (2.8), (5.1) and (5.3), it remains to show that

$$
\int_{0}^{\infty} e^{-t(a+x / 2)} f(t, x) d t=\sum_{m=0}^{N-1} \frac{C_{m}(x)}{(a+x / 2)^{m+1}}+0\left(x^{-1-r-N / 2}\right) .
$$

By (2.4) and (2.8),

$$
f(t, x) \ll e^{x(1-w+t / 2)} t^{-1} w^{s}(w x)^{-r},
$$

and so

$$
e^{-t(a+x / 2)} f(t, x) \ll e^{-a t} e^{x(1-w)} x^{-r} t^{s-1}
$$

uniformly for $t \geqq 1$. Since 


$$
1-w<-1 / 2 \text { for } t \geqq 1
$$

it follows from (5.8) that

$$
\int_{1}^{\infty} e^{-t(a+x / 2)} f(t, x) d t \ll e^{-x / 2} x^{-r} \int_{1}^{\infty} e^{-t \operatorname{Re}(a)} t^{\operatorname{Re}(s)-1} d t \ll e^{-x / 2}
$$

In view of (5.6) and (5.10), it remains to show that

$$
\int_{0}^{1} e^{-t(a+x / 2)} f(t, x) d t=\sum_{m=0}^{N-1} \frac{C_{m}(x)}{(a+x / 2)^{m+1}}+0\left(x^{-1-r-N / 2}\right) .
$$

Integrating by parts $N$ times, we obtain

$$
\begin{aligned}
\int_{0}^{1} e^{-t(a+x / 2)} f(t, x) d t= & \sum_{m=0}^{N-1} \frac{f^{(m)}(0, x)-f^{(m)}(1, x) e^{-(a+x / 2)}}{(a+x / 2)^{m+1}} \\
& +(a+x / 2)^{-N} \int_{0}^{1} e^{-t(a+x / 2)} f^{(N)}(t, x) d t
\end{aligned}
$$

By Lemma 4,

$$
e^{-(a+x / 2)} f^{(m)}(1, x) \ll e^{-(a+x / 2)} x^{3 m / 2} \ll e^{-x / 3} .
$$

Thus, to prove $(5.11)$, it remains to prove that

$$
\int_{0}^{1} e^{-t(a+x / 2)} f^{(N)}(t, x) d t=0\left(x^{N / 2-r-1}\right)
$$

Again by Lemma 4,

$$
\begin{aligned}
\int_{0}^{1} e^{-t(a+x / 2)} f^{(N)}(t, x) & \ll x^{N / 2-r} \int_{0}^{1} e^{-t \operatorname{Re}(a+x / 2)} \sum_{j=0}^{N}|x t|^{j} d t \\
& \ll x^{N / 2-r} \sum_{j=0}^{N}|x|^{j} \int_{0}^{\infty} e^{-t \operatorname{Re}(a+x / 2)} t^{j} d t \\
= & x^{N / 2-r} \sum_{j=0}^{N} \frac{|x|^{j} j !}{(\operatorname{Re}(a+x / 2))^{j+1}} \\
& \ll x^{N / 2-r} \sum_{j=0}^{N} \frac{|x|^{j} j !}{|x|^{j+1} \ll x^{N / 2-r-1}}
\end{aligned}
$$




\section{Asymptotic expansion of $C_{m}(x)$}

As promised following Corollary 3 , we show here that $C_{m}(x)$ possesses an asymptotic expansion in descending powers of $x$.

As in Section 4, we will estimate $C_{m}(x)=f^{(m)}(0, x)$ by combining Leibniz's rule with formulas for the $n$th derivatives of $(-t)^{r-1}, w^{s}, e^{x(1-w+t / 2)}$, and $U(r, s+r ; w x)$. The $n$th derivatives of $(-t)^{r-1}$ and $w^{s}$ at $t=0$ are constants. Since the function $g(t)$ in (4.10) satisfies $g(0)=0$, the $n$th derivative of $e^{x(1+t / 2-w)}$ at $t=0$ is, by $(4.10)$, a polynomial in $x$. It remains to show that the $n$th derivative of $U(r, s+r ; w x)$ at $t=0$ has an asymptotic expansion in descending powers of $x$. By (4.1) with $h(z)=U(r, s+r ; z)$ and $g(t)=w x$, we have

$$
\left.\frac{d^{n}}{d t^{n}} U(r, s+r ; w x)\right|_{t=0}=\left.\sum_{k=0}^{n} E_{k} x^{k} \frac{d^{k}}{d z^{k}} U(r, s+r ; z)\right|_{z=x}
$$

for some constants $E_{k}$. Using the asymptotic formula (4.8) in (6.1), we obtain the desired result.

If $s$ is a positive integer, the stronger result holds that $C_{m}(x)$ is a Laurent polynomial. To see this, note that when $s$ is an integer,

$$
U(r, s+r ; z)=\sum_{k=0}^{s-1} \frac{(-1)^{k}(r)_{k}(1-s)_{k}}{z^{k+r}}
$$

by (2.5) with $r$ and $s$ interchanged. Thus, $U(r, s+r ; z)$ and its derivatives with respect to $z$ are Laurent polynomials in $z$, and the result follows from (6.1) as before.

\section{REFERENCES}

1. M. Abramowitz and I. Stegun, Handbook of Mathematical Functions (Dover, New York, 1972).

2. B. C. Berndt, Ramanujan's Notebooks, Part V (Springer-Verlag, New York), to appear.

3. A. Erdélyl, ed., Higher Transcendental Functions, Vol. II (McGraw-Hill, New York, 1953).

4. N. N. Lebedev, Special Functions and their Applications (Dover, New York, 1972).

5. F. W. J. Olver, Asymptotics and Special Functions (Academic Press, New York, 1974).

6. S. Ramanujan, Notebooks (2 volumes) (Tata Institute of Fundamental Research, Bombay, 1957).

7. J. Riondan, An Introduction to Combinatorial Analysis (Princeton University Press, Princeton, New Jersey, 1978).

8. S. Roman, The formula of Faa di Bruno, Amer. Math. Monthly 87 (1980), 805-809.

Department of Mathematics

1409 West Green Street

UNIVERSITY OF ILLINOIS

URBANA, IL 61801
Department of Mathematics University of California, San Diego LA Jolla, CA 92093-0112 